\title{
EVALUATION OF DRIVING-ASSISTANCE SYSTEMS BASED ON DRIVERS' WORKLOAD
}

\author{
Yuji Takada and Osamu Shimoyama \\ Vehicle Research Laboratory, Nissan Research Center \\ Nissan Motor Co., Ltd. \\ Yokosuka, Kanagawa, Japan \\ E-mail: taka-yuji@mail.nissan.co.jp
}

\begin{abstract}
Summary: This paper describes an experimental study concerning an evaluation of advanced driving-assistance systems using methods for estimating workload levels. The effects of such systems on drivers' mental workload and driving performance were measured experimentally using the driving simulator. Six subjects were instructed to drive the simulator in a highway environment with and without Adaptive Cruise Control (ACC) and/or the collision-warning system (CWS). To assess the effectiveness of these systems on drivers' performance, the subjects were asked to calculate sums of single- or double-digit figures displayed. The results show that higher accuracy was obtained under a condition with ACC than without it. To estimate the subjects' mental workload levels, their electrocardiograms and respiration data were recorded during the sessions and the RRI, heart rate variance and respiration frequency were calculated. The results indicate that the provision of the CWS and ACC reduced the subjects’ mental workload compared with the situation without the systems.
\end{abstract}

\section{INTRODUCTION}

Driving-assistance systems for helping drivers with the operation of their vehicles are beginning to find practical application. The purpose for researching and developing such systems is to improve user convenience by reducing the mental and physical workload involved in driving a vehicle. Many studies have been conducted so far that have focused on drivers' mental workload (Stanton, 1998); attempts have been made to analyze the impact of such systems on drivers by using methods for estimating their mental workload in terms of performance indices (Matthews, 1998), physiological indices and subjective ratings. It has been pointed out that each of these methods, though, has its advantages and disadvantages and that there are limits to their detectable range.

In this research, therefore, an attempt was made to evaluate the effects of driving-assistance systems on drivers' mental workload from a multidimensional perspective by applying several evaluation methods simultaneously. A driving simulator was used in the experiments, and a collision-warning system (CWS) and Adaptive Cruise Control (ACC) was selected for evaluation. 


\section{EXPERIMENTS}

\section{Experimental Apparatus}

Figure 1 shows the appearance of the driving simulator (DS), which was fitted with a motordriven motion base having six coordinated degrees of freedom. On the inside, a 6” TFT liquid crystal display and a numeric keypad are located in the center of the dashboard (Fig. 2).

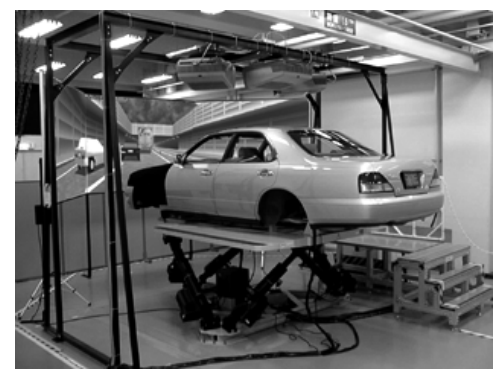

Figure 1. Motion-based simulator

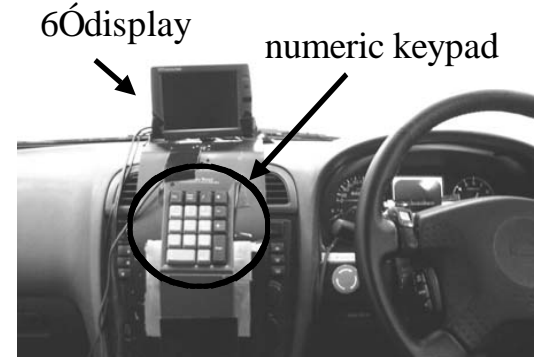

Figure 2. Experimental equipment

\section{Driving Scenarios}

The subjects were instructed to follow a preceding vehicle in the same lane of an expressway. One driving session took 20 minutes to complete. To emulate the real world, a scenario of deceleration and acceleration by the preceding vehicle was repeated eight times. Another scenario involving cutting in by a third vehicle and subsequent deceleration was also repeated eight times.

\section{Experimental Conditions}

Subjects. The subjects were six males with an average age of 39. They had experienced driving both an actual vehicle and the DS fitted with a CWS and ACC.

Test tasks. The subjects were instructed that their main task was to drive the DS without colliding with the vehicle ahead. Moreover, they were also given a sub-task of adding two single-digit numbers from 0 to 9 or adding two double-digit numbers from 10 to 49. In these addition tasks, the numbers were presented on the 6" display, and the subjects entered the answer via the numeric keypad. The addition equation was displayed for $5 \mathrm{~s}$ and the answer was also supposed to be input within $5 \mathrm{~s}$. Addition tasks were presented approximately every $400 \mathrm{~m}$.

Driving-assistance systems. The headway distance for issuing a collision warning was calculated in accordance with the general equation shown below.

$$
D=V_{1} \times T+V_{1}^{2} / 2 a_{1}-V_{2}^{2} / 2 a_{2}
$$

where $D$ is the warning distance, $T$ is the dead time, $V_{1}$ and $V_{2}$ indicate the respective velocity and $a_{1}$ and $a_{2}$ indicate the respective deceleration of the host vehicle and the vehicle ahead. In these experiments, $T$ was set at $0.8 \mathrm{~s}$, and deceleration $a_{1}$ and $a_{2}$ were both set at $0.35 \mathrm{G}$. Under ACC driving, the cruising velocity of the host vehicle was set at $100 \mathrm{~km} / \mathrm{h}$ and the headway distance was set to equal $1.5 \mathrm{~s}$. The host vehicle was accelerated or decelerated automatically so as to follow the vehicle in front. However, the system did not produce 
deceleration above a given level. Accordingly, when the vehicle ahead decelerated greatly, the subjects had to brake manually. The collision warning system was designed to issue a warning during ACC operation.

\section{Measured Items}

Performance index. Time histories of the subjects' keypad-entered responses to the addition tasks were recorded. The rate of correct answers to the addition tasks and the time to finish inputting answer were found for each subject.

Physiological indices. Electrocardiograms and respiration rates were measured for the subjects in all the driving sessions. The R-R interval (RRI) and the variance in RRI (RRV) were found from the recorded electrocardiograms. The RRI and RRV indices tend to show relatively lower values as the mental workload of test subjects increases (Mouri, 1994). In this study, therefore, RRI and RRV for each type of addition task during CWS driving and ACC driving were found as a ratio of RRI and RRV during manual driving as the baseline. For example, the ratio of $\mathrm{RRI}_{\mathrm{acc}}$ during ACC driving to $\mathrm{RRI}_{\text {manual }}$ during manual driving was found as

$$
\text { Ratio }=R R I_{\text {acc }} / R R I_{\text {manual }}
$$

Using this ratio, an expression such as ratio $>1.0$ signifies that the mental workload with a driving-assistance system is lower than that for manual driving.

A fast Fourier transform (FFT) frequency analysis was also performed on the RRI data to calculate the power spectrum of heart rate variance. The peak that appears around $0.1 \mathrm{~Hz}$ is referred to as the low-frequency (LF) component of heart rate variance and tends to show a relatively small value under a condition of a high mental workload (Aasman, 1987). Accordingly, the ratio of the LF component during manual driving to the LF component during CWS and ACC driving was also calculated. In this case, when the ratio shows a value greater than one, it can be concluded that the mental workload with a driving-assistance system is lower than that during manual driving.

It is known that the respiration frequency rise when concentration is required and when there is a feeling of time pressure. Therefore, the respiration frequency was calculated by FFT on the respiration data. In addition, the respiration frequency for each type of addition task during CWS driving and ACC driving was found as a ratio of that during manual driving as the baseline. In this case, when the ratio shows a value smaller than one, it can be concluded that the mental workload with a driving-assistance system is lower than that during manual driving.

\section{Experimental Procedure}

Each subject performed driving sessions under the nine combinations of addition sub-tasks and driving-assistance systems shown in Table 1. Experiments were carried out over three days. 
Table 1. Experimental conditions

\begin{tabular}{cllc}
\hline No. & assistance system & additional task & day \\
\cline { 1 - 2 } 1 & none(manual) & none & first \\
\hline 2 & CWS & & \\
\hline 3 & ACC & 1-digit numbers & second \\
\hline 4 & none(manual) & & \\
\hline 5 & CWS & 2-digit numbers & third \\
\hline 6 & ACC & & \\
\hline 7 & none(manual) & CWS & \\
\hline 9 & ACC &
\end{tabular}

\section{EXPERIMENTAL RESULTS}

\section{Performance Index}

The average and standard deviation were calculated for all the subjects and the results are shown in Fig. 3. In Fig. 3-(a), it is seen that the rate of correct answers tended to increase in the order of manual driving, CWS driving and ACC driving. A significant difference $(\mathrm{p}<0.05)$ between manual driving and ACC driving was observed for the performance of single-digit addition tasks. On the other hand, the results in Fig. 3-(b) indicate that there was no striking difference in answering time between the two types of driving-assistance systems.

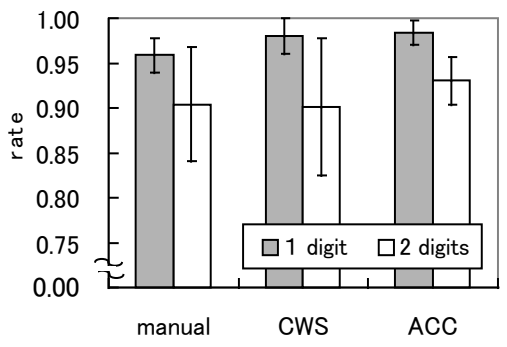

(a) Rate of correct answers

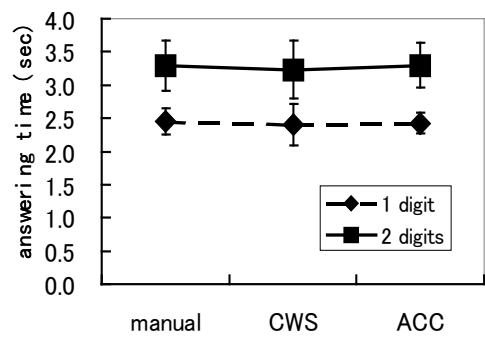

(b) Answering time

Figure 3. Results of addition tasks 


\section{Physiological Indices}

$R R I$ and $R R V$ ratios. the average and standard deviation were calculated for all the subjects and the results are shown in Fig. 4. It is seen in Fig. 4-(a) that the RRI average was nearly the same for manual driving and CWS driving and that the mental workload was reduced during ACC driving. The results in Fig. 4-(b) show that the mental workload was lower during CWS driving and ACC driving without any addition tasks and during CWS driving with double-digit addition tasks.

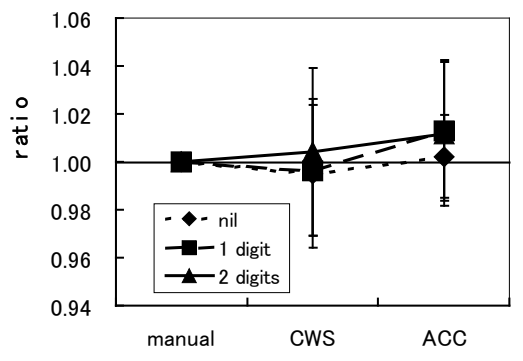

(a) RRI

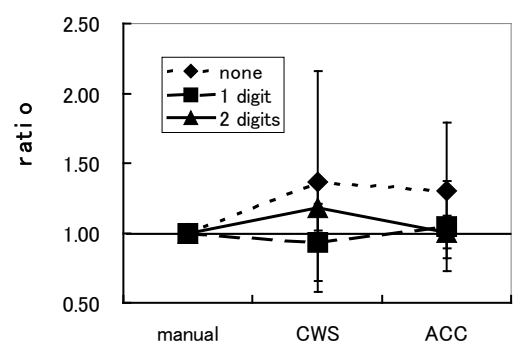

(b) RRV

Figure 4. Ratios of (a)RRI and (b)RRV, manual to driving-assistance systems

LH ratios. The average and standard deviation were found for all the subjects and the results are shown in Fig. 5. Although some data dispersion is seen among the subjects in this figure, a comparison of the average values indicates that the mental workload levels for single-digit addition tasks during CWS driving and for double-digit addition tasks during ACC driving were the same as

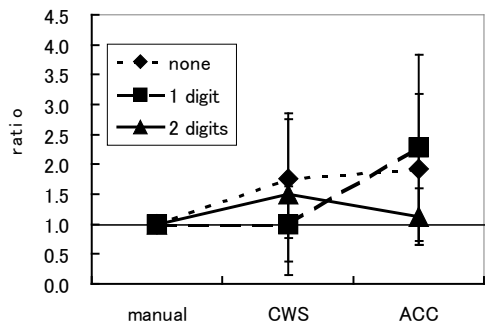

Figure 5. Ratios of power density in LF band, manual to driving-assistance systems

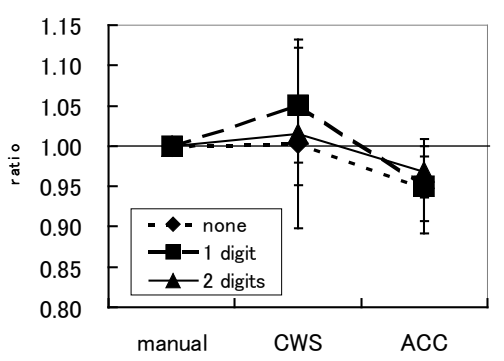

Figure 6. Ratios of respiration frequency, manual to driving-assistance systems

during manual driving. Under the other conditions, the mental workload levels were lower during both CWS driving and ACC driving.

Respiration ratios. The results are shown in Fig. 6. It is seen that the respiration frequency was higher for both types of addition tasks during CWS driving than it was during manual driving, whereas it was lower for both types of addition tasks during ACC driving. Therefore, based on the results of this analysis, it can be estimated that the mental workload increased under CWS driving and decreased under ACC driving.

\section{DISCUSSION}

The experimental conditions were then arranged in order from the lowest to the highest mental workload, based on the average scores calculated for the subjects with the different evaluation 
methods used in this study. The results are shown in Table 2. The same rating in the table means that the averages were virtually the same.

It can be concluded from the overall results given in the table that ACC-based vehicle control has the effect of reducing drivers' mental workload. The support provided by a collision warning system tends to differ greatly from one driver to another, though it has an effect on reducing the mental workload compared with the level seen for ordinary driving.

Table 2. Comparison of results

\begin{tabular}{|c|c|c|c|c|c|c|c|c|c|c|}
\hline \multirow{2}{*}{\multicolumn{2}{|c|}{ evaluation methods task }} & \multicolumn{3}{|c|}{ none } & \multicolumn{3}{|c|}{1 digit } & \multicolumn{3}{|c|}{2 digits } \\
\hline & & Manual & CWS & ACC & Manual & CWS & ACC & Manual & CWS & $\mathrm{ACC}$ \\
\hline \multirow[t]{2}{*}{ performance } & answering time & - & - & - & 1 & 1 & 1 & 1 & 1 & 1 \\
\hline & correct answer & - & - & - & 3 & 2 & 1 & 2 & 1 & 1 \\
\hline \multirow[t]{5}{*}{ physiological } & RRI & 1 & 2 & 1 & 2 & 3 & 1 & 3 & 2 & 1 \\
\hline & RRV & 3 & 1 & 2 & 2 & 3 & 1 & 2 & 1 & 2 \\
\hline & LF & 3 & 2 & 1 & 2 & 2 & 1 & 2 & 1 & 2 \\
\hline & Respiration & 2 & 2 & 1 & 2 & 3 & 1 & 2 & 3 & 1 \\
\hline & average & 2.25 & 1.75 & 1.25 & 2.00 & 2.33 & 1.00 & 2.00 & 1.5 & 1.33 \\
\hline
\end{tabular}

\section{CONCLUSIONS}

The effects of driving-assistance systems, collision warning system and ACC, on drivers' mental workload were evaluated comprehensively using a performance index, physiological indices. The major conclusions drawn from this evaluation are summarized below.

(1) ACC is effective in reducing drivers' mental workload and it also has an effect on improving operational performance.

(2) The effect of a collision warning system shows large individual differences. However, its effect on reducing drivers' mental workload has been confirmed.

\section{REFERENCES}

Aasman, J. 1987, Effort and the Measurement of Heart-Rate Variability, Human Factors, 29(2), 161-170

Mattews, G. 1998, Driver Stress and Performance on a Driving Simulator, Human Factors, 40(1), 136-149

Mouri, H. 1994, An investigation of driver stress induced by vehicle handling characteristics, JSAE Review, 15, 235-26

Stanton, N.A. 1998, Vehicle automation and driving performance, Ergonomics, 41(7), 10141028 